\title{
Application of 16-State TCM-UGM and TCM for Improving the Quality of Compressed Color Image Transmission
}

\author{
Benaïssa Mohamed \\ Department of Electrical Engineering Faculty of the sciences and technology \\ Bechar University, B.P 417 Bechar (08000), Algeria \\ E-mail: moh.benaissa@gmail.com \\ Bassou Abdesselam \\ Department of Electrical Engineering Faculty of the sciences and technology \\ Bechar University, B.P 417 Bechar (08000), Algeria \\ E-mail: a.bassou@gmail.com \\ Beladgham Mohammed \\ Department of Electrical Engineering Faculty of the sciences and technology \\ Bechar University, B.P 417 Bechar (08000), Algeria \\ E-mail: beladgham.tlm @gmail.com
}

Taleb-Ahmed Abdelmalik

Laboratory of automatics, Mechanical and computer Science, Valenciennes University, France

E-mail: taleb@univ-valenciennes.fr

Moulay Lakhdar Abdelmounaim

Department of Electrical Engineering Faculty of the sciences and technology Bechar University, B.P 417 Bechar (08000), Algeria

E-mail: Moulaylakhdar78@yahoo.fr

\begin{abstract}
The aim of this paper is to investigate the quality of transmitted color images using 16-state TCMUGM or TCM channel code over Rayleigh fading channel. Considering SPIHT-based compression algorithm and image quality metrics (IQMs), the simulation results for throughput of $2 \mathrm{bit} / \mathrm{s} / \mathrm{Hz}$, showed that the communication system using TCM-UGM allows better performance compared to TCM and better protects the compressed color image during transmission. For transmission tests compressed colors images, the TCMUGM system outperforms the performance of the TCM by $3 \mathrm{~dB}$ at BER $=10^{-5}$ and $4.59 \mathrm{~dB}$ at FER $=3.10^{-3}$. For example, for Lena color image, the 16-state TCM-UGM system gives best performance that the 16-state TCM system. The gain is the $5.02 \mathrm{~dB}$ and $17.90 \%$ for the PSNR and MMSIM respectively.
\end{abstract}

Index Terms-TCM-UGM, TCM, compressed color image, SPIHT.

\section{INTRODUCTION}

The trellis-coded modulation (TCM) was introduced by Ungerboeck in 1982 [1]. This coding technique improves error performance of synchronous data links without sacrificing data rate or requiring more bandwidth. This is achieved by channel coding with expanded sets of multilevel/phase signals in a manner which increases free Euclidean distance. The TCM codes can be interpreted as binary convolutional codes with a mapping of coded bits into channel signals using mapping by set partitioning.

A new variant of the TCM modulation based on Ungerboeck mapping combined with Gray coding, called 'Trellis-Coded Modulation with Ungerboeck-Gray Mapping' (TCM-UGM) has been proposed by Bassou [2] Bassou and Djebbari have proposed this new schema for a spectral efficiency greater than or equal to $3 \mathrm{bits} / \mathrm{s} / \mathrm{Hz}$. The difference between these two structures resides in the positioning of the uncoded bits out in the encoder. Indeed, for TCM-UGM the uncoded bits are placed before the parity bit. The latter cannot in any way represent the MSB or the LSB of the transmitted symbol. In the absence of uncoded bits, the TCM-UGM is equivalent to the proposed Ungerboeck TCM.

A variant of the TCM-UGM for spectral efficiency of 2 bits $/ \mathrm{s} / \mathrm{Hz}, 8 \mathrm{PSK}$ modulation and rate equal to $2 / 3$ is proposed by Benaïssa et al. [3] for transmit the gray image. The suggestion is do not use the non-coded bit necessary, and to replace it with one of the two systematic bits used for the generation of the parity bit. 
Simulation results for spectral efficiency $2 \mathrm{bits} / \mathrm{s} / \mathrm{Hz}$, rate $2 / 3$, shown that TCM-UGM outperforms the original TCM scheme proposed by Ungerboeck over Rayleigh fading channel. In this paper this new scheme is used for the transmission of compressed color image. Image compression is a vital task for image transmission and storage. Many compression schemes by transformation have been proposed, we can cite the standards JPEG images, MPEG 1 and 2 for video compression. All of these standards are based on the discrete cosine transform (DCT)[4]. Over the past ten years, the wavelets (DWT) have had a huge success in the field of image processing, and have been used to solve many problems such as image compression [5]. One of the most successful practical image coders today based on wavelet transforms for the noiseless channel was originally developed by Shapiro [6] and later refined by Said and Pearlman [7]. In [7], a wavelet-based still image coding algorithm known as set partitioning in hierarchical trees (SPIHT) is developed that generates a continuously scalable bitstream. This means that a single encoded bitstream can be used to produce images at various bit-rates and quality, without any drop in compression. These wavelet-based encoders have come out to perform better than almost any other existing compression scheme. In addition, they are progressive and computationally simple.

In this article, a comparison between the TCM and the new variant the TCM-UGM for $2 \mathrm{bits} / \mathrm{s} / \mathrm{Hz}$ is proposed for transmit the coded color image based on Set Partitioning in Hierarchical Trees transmission over Rayleigh channel. In section II, the TCM-UGM for 2 bit/s/Hz, is introduced. In section III, the SPIHT Coding Scheme is described. In section IV the proposal system is exposed. The Image Quality Metrics are mentioned in the section $\mathrm{V}$. The simulation results are presented in section VI. Finally, the paper is concluded in section VII.

\section{TCM-UGM FOR 2 BITS/S/HZ}

The TCM scheme, proposed by Ungerboeck [1], was designed for spectral efficiency of $\mathrm{m} \mathrm{bits} / \mathrm{sec} / \mathrm{Hz}$, where $\mathrm{m}$ bits are input to the encoder (among input bits $\tilde{m} \geq 0$ are uncoded) and $m+1$ bits are output and mapped with $2^{m+1}$-ary modulation using set partitioning yielding a coding rate

$$
R=\frac{m}{m+1}
$$

In this case the mapping by set partitioning (called also Ungerboeck mapping) is applied. The Ungerboeck TCM encoder was chosen by maximizing $d_{f}$. In [1], $d_{f}$ is compute by an algorithm that replaces the search in TCM trellis for the path that maximizes $d_{f}$.

In [2], the TCM-UGM scheme considered, for a spectral efficiency of $\mathrm{bits} / \mathrm{sec} / \mathrm{Hz}$, that the mapper is a $2^{m+1}$-airy that uses a mapping technique combining Ungerboeck mapping and Gray code mapping.
The Ungerboeck coding is applied only to the $m-\tilde{m}$ bits used to generate the parity bit. The $\tilde{m}$ uncoded bits and the parity bit use Gray coding. for apply the two types of coding in the TCM-UGM the number of systematic bit must be greater than one and the number of uncoded bit must be greater or equal to one, implying a minimum spectral efficiency of 3 bits/s/Hz[2]. When no uncoded bits are considered $(\tilde{m}=0)$, this scheme is equivalent to Ungerboeck TCM scheme with $\tilde{m}=0$.

In [3] a variant of the TCM-UGM for spectral efficiency of $2 \mathrm{bits} / \mathrm{s} / \mathrm{Hz}, 8 \mathrm{PSK}$ constellation and rate equal to $2 / 3$ is proposed. The suggestion is do not use the uncoded bit necessary, and to replace it with one of the two systematic bits used for the generation of the parity bit. The variant of TCM-UGM encoder, is illustrated in Fig. 1, for two input bits $(m=2)$, without uncoded bits $(\tilde{m}=0)$. The 8PSK constellation used for the TCMUGM encoder is shown in Fig. 2 [3].

The performance simulation of the TCM and TCMUGM for the optimal code-generator (table 1) [3][8], using 8PSK Ungerboeck mapper (for TCM) and Ungerboeck-Gray mapper (for TCM-UGM) for spectral efficiency $2 \mathrm{bit} / \mathrm{s} / \mathrm{Hz}$, rate $2 / 3$ and 16 and 32-state TCM or TCM-UGM encoder over Rayleigh channel illustrated that, for 16 and 32 state, the TCM and the TCM-UGM presents approximately the same performance in terms of BER, for the low $E_{b} / N_{0}(<11 \mathrm{~dB})$. TCM-UGM has better performance at high $E_{b} / N_{0}$. With 16 and 32-state, the TCM-UGM outperforms the TCM by $3.78 \mathrm{~dB}$ and $3.98 \mathrm{~dB}$ respectively at $\mathrm{BER}=10^{-5}$. Simulation results also showed that for the TCM-UGM with 16-state outperforms the TCM with 32-states of $2.8 \mathrm{~dB}$ at $\mathrm{BER}=10^{-5}$. In this paper we consider only the simulation for 16-states for transmit the coded Image color based on Set Partitioning in Hierarchical Trees.

\section{SPIHT CODING SCHEME}

In the SPIHT algorithm, the image is first decomposed into a number of subbands by means of hierarchical wavelet decomposition [9]. For example, the subbands obtained for a three-level decomposition are shown in Fig. 3. The subband coefficients are then grouped into sets known as spatial-orientation trees, which efficiently exploit the correlation between the frequency bands. The coefficients in each spatial orientation tree are then progressively coded from the most significant bit-planes (MSB) to the least significant bit-planes (LSB), starting with the coefficients with the highest magnitude and at the lowest pyramid levels. The SPIHT multistage encoding process employs three lists and sets:

1. The list of insignificant pixels (LIP) contains individual coefficients that have magnitudes smaller than the threshold.

2. The list of insignificant sets (LIS) contains sets of wavelet coefficients that are defined by tree structures and are found to have magnitudes smaller than the threshold (insignificant). The sets exclude the 
coefficients corresponding to the tree and all subtree roots and they have at least four elements.

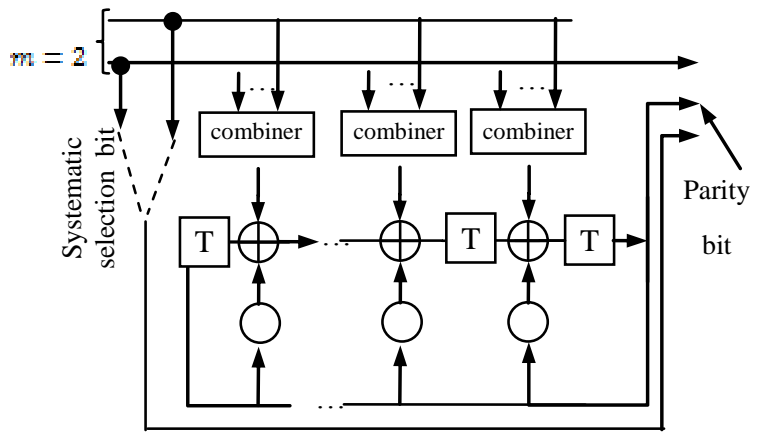

Fig.1: New variant of TCM-UGM for 2 bits/s/Hz

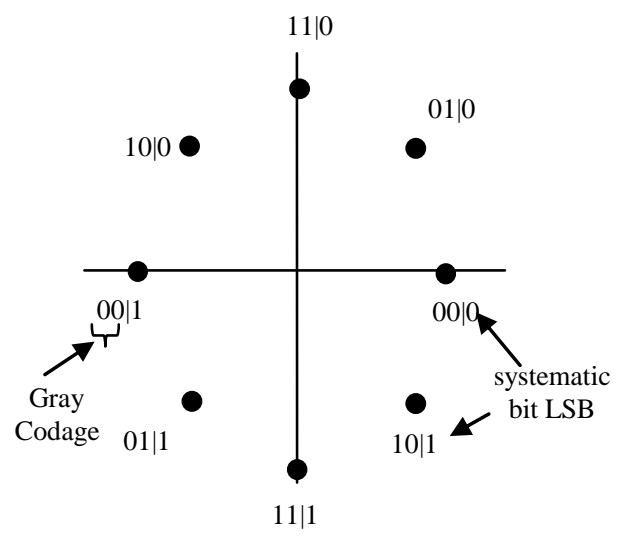

Fig. 2: TCM-UGM for a spectral efficiency of $2 \mathrm{bits} / \mathrm{s} / \mathrm{Hz}$

Table 1: Code-Generator for Spectral efficiency $2 \mathrm{bits} / \mathrm{s} / \mathrm{Hz}$

\begin{tabular}{|l|c|c|ccc|}
\cline { 3 - 6 } \multicolumn{1}{c|}{} & \multirow{2}{*}{$\boldsymbol{d}_{\boldsymbol{f}}{ }^{2}$} & \multirow{2}{*}{$\begin{array}{l}\text { Memory } \\
\text { order }\end{array}$} & \multicolumn{4}{|c|}{ Code-generator } \\
\cline { 4 - 6 } & & & $\mathbf{h}_{\mathbf{0}}$ & $\mathbf{h}_{\mathbf{1}}$ & $\mathbf{h}_{\mathbf{2}}$ \\
\hline TCM & 5.172 & 4 & 31 & 14 & 30 \\
\hline TCM-UGM & 5.172 & 4 & 23 & 34 & 15 \\
\hline TCM & 5.758 & 5 & 51 & 70 & 34 \\
\hline TCM-UGM & 5.758 & 5 & 51 & 34 & 05 \\
\hline
\end{tabular}

3. The list of significant pixels (LSP) is a list of pixels found to have magnitudes larger than the threshold (significant).

4. The set of offspring (direct descendants) of a tree node, $O(i, j)$, in the tree structures is defined by pixel location $(i, j)$. The set of descendants, $D(i, j)$, of a node is defined by pixel location $(i, j) . L(i, j)$ is defined as $L(i, j)=D(i, j)-O(i, j)$. The threshold, $T$, for the first bit-plane is equal to $2^{\mathrm{n}}$, and $n=\left[\log _{2}\left(\max _{(i, j)}\{c(i, j)\}\right)\right]$
Where $c(i, j)$ represents the $(i, j)^{t h}$ wavelet coefficient. All the wavelet coefficients are searched in order to obtain the maximum $c(i, j)$ after executing the discrete wavelet transform. For operations in the subsequent bitplanes of threshold $T, n$ is reduced by 1 . For each pixel in the LIP, one bit is used to describe its significance. If it is not significant, the pixel remains in the LIP and no more bits are generated; otherwise, a sign bit is produced and the pixel is moved to the LSP. Similarly, each set in the LIS requires one bit for the significance information. The insignificant sets remain in the LIS; the significant sets are partitioned into subsets, which are processed in the same manner and at the same resolution until each significant subset has exactly one coefficient. Finally, each pixel in the LSP is refined with one bit. The abovementioned procedure is then repeated for the subsequent resolution.

In this article the biorthogonal wavelet $5 / 3$ is used. These wavelets are part of the family of symmetric biorthogonal wavelet CDF. The wavelets $5 / 3$ have a great number of null moments for a relatively short support. They are more symmetrical and very close to orthogonality. This is an important feature in coding which ensures that the reconstruction error is very close to the quantization error in terms of mean squared error. Antonini and Barlaud were the first [9] to show the superiority of the biorthogonal wavelet transform $5 / 3$ for the decorrelation of natural images. It has been widely used in image coding [7]-[10] and is used by the JPEG2000 codec [11]. The bit stream produced by the set partitioning in hierarchical trees (SPIHT) image color coding algorithm is transmitted by the TCM or TCMUGM.

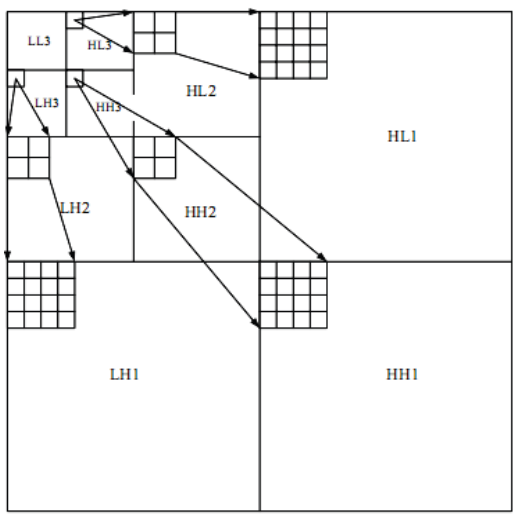

Fig. 3: 3-level wavelet decomposition and spatial orientation tree

\section{PROPOSAL SYSTEM}

Fig. 4 represents the image transmission system for which we evaluate the BER, FER and two Image Quality Metrics (IQMs) after decoding.

Before applying Wavelet transform on the color image, the RGB color images are converts into $\mathrm{YCbCr}$ form, and 
then applying Wavelet transform on each layer independently, this means each layer from $\mathrm{YCbCr}$ are compressed as a grayscale image. $\mathrm{YCbCr}$ refers to the color resolution of digital component video signals, which is based on sampling rates. In order to compress bandwidth, $\mathrm{Cb}$ and $\mathrm{Cr}$ are sampled at a lower rate than $\mathrm{Y}$, which is technically known as "chroma subsampling."

This means that some color information in the image is being discarded, but not brightness (luma) information. We obtains the best rate of compression using the rich less layer for the chromatic composante $\mathrm{Cb}$ and $\mathrm{Cr}$.

$$
\left\{\begin{array}{l}
Y=0.2989 * R+0.5866 * G+0.1145 * B \\
C b=-0.1687 * R-0.3312 * G+0.5 * B \\
C r=0.5 * R-0.4183 * G-0.0816 * B
\end{array}\right.
$$

When the decomposition image is obtained, we try to find a way to code the Wavelet transform into an efficient result, taking redundancy and storage space into consideration. After, we apply SPIHT algorithm on each layer $(\mathrm{Y}, \mathrm{Cr}, \mathrm{Cb})$ independently .

The SPIHT (Y,Cr,Cb) generates a continuously scalable bit stream. The bit stream resulting constitute the sequence of data to be transmitted. The information data is first encoded by the TCM or the variant TCM-UGM encoder exposed in section 2. The complex constellation symbols, generated by mapper, are interleaved and transmitted over the Rayleigh channel. At the receiver end, the received symbols are decoded by the TCM or the TCM-UGM decoder and are used for the reconstruction image.

Both compression and transmission errors may degrade the quality of images. Over the years, a number of researchers have contributed significant research in the design of full reference image quality assessment algorithms, claiming to have made headway in their respective domains [12]. In this study, two Image Quality Metrics (IQMs), the Peak signal-to-noise ratio (PSNR) and the Multi-scale structural similarity (MMSIM) were employed in the image quality assessment.

\section{IMAGE QUALITY METRICS}

The PSNR is the most commonly used as a measure of quality of reconstruction in image compression. The PSNR were identified using the following formulate:

$$
M S E=\frac{1}{M \cdot N} \cdot \sum_{i=0}^{M-1} \sum_{j=0}^{-1}[I(i, j)-\hat{I}(i, j)]^{2}
$$

Mean Square Error (MSE) which requires two MxN gray scale images $I$ and $\hat{I}$ where one of the images is considered as a compression of the other is defined as:

- The PSNR is defined as:

$$
P S N R=10 \cdot \log _{10} \frac{\left(2^{R}-1\right)^{2}}{M S E}[d B]
$$

Usually an image is encoded on 8 bits. It is represented by 256 gray levels, which vary between 0 and 255, the extent or dynamics of the image is 255 .

PSNR of a color image (RGB) is defined by the equation:

$$
P S N R=10 \log _{10} \frac{255^{2} \times 3}{\operatorname{MSE}(R)+\operatorname{MSE}(G)+\operatorname{MSE}(B)}
$$
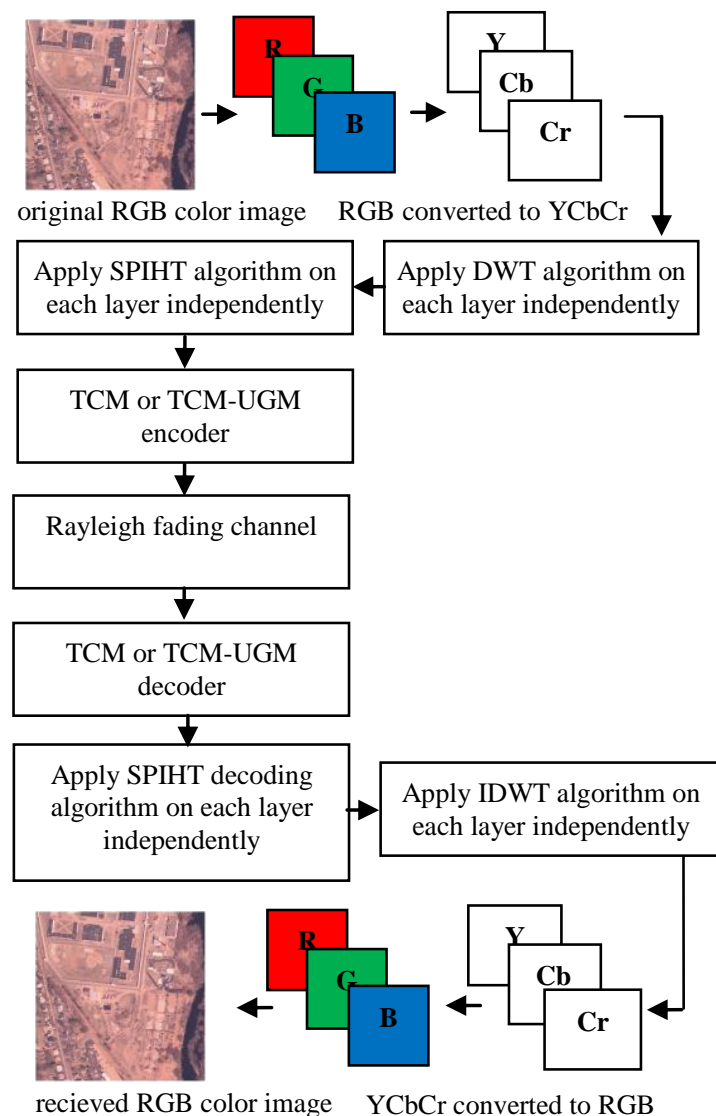

Fig. 4: System overview

- $\quad$ The structural similarity index (SSIM):

The PSNR measurement gives a numerical value on the damage, but it does not describe its type. Moreover, as is often noted in [13], [14], it does not quite represent the quality perceived by human observers. For medical imaging applications where images are degraded must eventually be examined by experts, traditional evaluation remains insufficient. For this reason, objective approaches are needed to assess the medical imaging quality [15]. We then evaluate a new paradigm to estimate the quality of medical images, specifically the ones compressed by wavelet transform, based on the 
assumption that the human visual system (HVS) is highly adapted to extract structural information. The similarity compares the brightness, contrast and structure between each pair of vectors, where the structural similarity index (SSIM) between two signals $\mathrm{x}$ and $\mathrm{y}$ is given by the following expression:

$$
\operatorname{SSIM}(x, y)=l(x, y) c(x, y) s(x, y)
$$

Finally the quality measurement can provide a spatial map of the local image quality, which provides more information on the image quality degradation, which is useful in medical imaging applications. For application, we require a single overall measurement of the whole image quality that is given by the following formula:

$$
\operatorname{MSSIM}(I, \hat{I})=\frac{1}{M} \sum_{i=1}^{M} \operatorname{SSIM}\left(I_{i}, \hat{I}_{i}\right)
$$

Where $I$ and $\hat{I}$ are respectively the reference and degraded images, $I_{i}$ and $\hat{I}_{i}$ are the contents of images at the i-th local window.

$M$ : the total number of local windows in image. The MSSIM values exhibit greater consistency with the visual quality.

\section{SIMULATION RESULT}

Our work is to compare the performance of the 16-state TCM and the 16-state TCM-UGM for the transmission of compressed color image. Therefore, it's important of reducing the bitrates of compression ( $R_{b}$ (number of bits per pixel)) for which the image quality remains acceptable. Estimates and judgments of the compressed image quality are given by the IQMs. First, we present the simulation results of the SPIHT algorithm on the three tests colors images: 'Lena', 'Concoraerial' and 'Celulle' of size $512 \times 512$ encoded by 8 bpp. Table. 2 shown below illustrates the Image Quality Metrics for different bit-rate values (number of bits per pixel). We note that the metric values increase with the bit rate of 0.1-1 bpp. Fig. 5 shown below illustrates the compressed image quality for different bitrates values for the test Cellule image. From $R_{b}=0.8$, we see, based on Table.2 (where the MMSIM is grant than 92\%) and Fig.6 that the image quality is very acceptable and will employ for the transmit to the proposed systems.

The performance of the encoding schemes is evaluated in terms of BER (bit error rate) and FER (frame error rate) versus bit energy to noise ratio $\left(E_{b} / N_{0}\right)$. The FER computation considers a frame length of 1024. Fig. 6 and Fig. 7, illustrate the performance in sense of BER and FER, respectively, of TCM and TCM-UGM for the transmission of test compressed colors images mentioned in table 2 for a $R_{b}=0.8$. It can be observed that the system TCM-UGM has better results than TCM system from a value of $10.7 \mathrm{~dB}$, for BER curves and it is always best for FER curves. The TCM-UGM system outperforms the performance of the TCM by $3 \mathrm{~dB}$ at BER $=10^{-5}$ and $4.59 \mathrm{~dB}$ at FER $=3.10^{-3}$.

The performance simulation over Rayleigh fading channel of the TCM and TCM-UGM are investigated for a spectral efficiency of $2 \mathrm{bits} / \mathrm{s} / \mathrm{Hz}$ for the three tests compressed colors images.

We apply the variant of the TCM-UGM and TCM for the three tests colors images with different values of $E_{b} / N_{0}$. Table 3 shows the variations of IQMs for a bitrates of $0.8 \mathrm{bpp}$. For Lena color image, the TCM-UGM system gives best performance that the TCM system. For the two IQMs, the gain is $5.02 \mathrm{~dB}$ and $17.90 \%$ for the PSNR and MMSIM respectively.

It can be observed that for all images, the TCM-UGM gives better results than the TCM. Fig. 8, 9 and 10, illustrate 'lena', 'Concordaerial' and 'Cellule', decompressed images, respectively noted in table 2 after the reception and the decompressed. Table 3 and Fig. 8, Fig. 9 and 10, illustrate the effectiveness of the proposed transmission system for the color image. These results show that the variant of 16-state TCM-UGM reduces transmission errors and better protects the compressed image during transmission.

\begin{tabular}{|c|c|c|c|c|c|c|c|}
\hline & & \multicolumn{6}{|c|}{$R_{b}$} \\
\hline & IQM & 0.1 & 0.2 & 0.4 & 0.6 & 0.8 & 1 \\
\hline \multirow{2}{*}{ 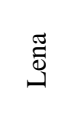 } & PSNR & 29.56 & 33.27 & 35.88 & 37.50 & 38.61 & 39.57 \\
\hline & MSSIM (\%) & 65.47 & 80.88 & 88.43 & 91.06 & 92.42 & 93.12 \\
\hline \multirow{2}{*}{ 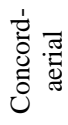 } & PSNR & 28.58 & 31.80 & 34.43 & 36.39 & 37.96 & 39.42 \\
\hline & MSSIM (\%) & 45.85 & 68.43 & 83.81 & 90.07 & 92.60 & 94.49 \\
\hline \multirow{2}{*}{$\frac{\frac{0}{\Xi}}{\dot{v}}$} & PSNR & 28.00 & 29.99 & 31.90 & 33.64 & 35.41 & 36.79 \\
\hline & MSSIM (\%) & 37.64 & 63.15 & 83.86 & 90.72 & 92.24 & 95.66 \\
\hline
\end{tabular}

Table.2: IQMs variation with Gall 5/3 and SPIHT coding 


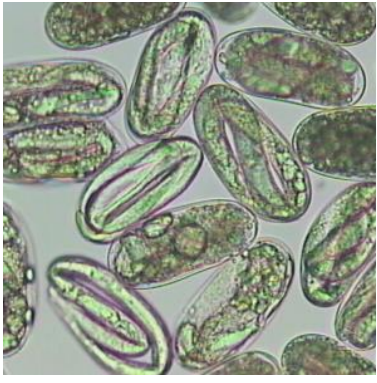

(a)

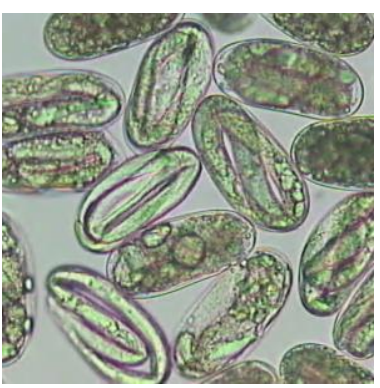

(c) (b)

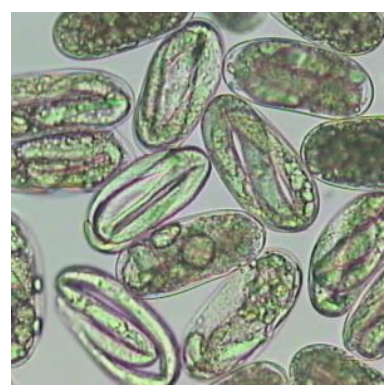

(d)

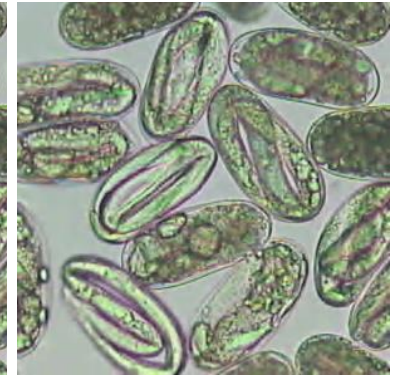

Table 3: IQMs variation vs $E_{b} / N_{0}$, (colors images tests)

\begin{tabular}{|c|c|c|c|}
\cline { 3 - 4 } \multicolumn{1}{c|}{} & TCM & TCM-UGM \\
\hline \multirow{4}{*}{ Lena } & $E_{b} / N_{0}$ & 21.25 & 18 \\
\cline { 2 - 4 } & PSNR & 33.22 & 38.24 \\
\cline { 2 - 4 } & MMSIM (\%) & 73.17 & 91.07 \\
\hline \multirow{4}{*}{$\begin{array}{c}\text { Concord- } \\
\text { aerial }\end{array}$} & $E_{b} / N_{0}$ & 21.5 & 18 \\
\cline { 2 - 4 } & PSNR & 30.54 & 35.08 \\
\cline { 2 - 4 } & MMSIM (\%) & 66.66 & 82.31 \\
\hline \multirow{3}{*}{\begin{tabular}{c} 
Cellule \\
\cline { 2 - 4 }
\end{tabular}} & $E_{b} / N_{0}$ & 21.5 & 18 \\
\cline { 2 - 4 } & PSNR & 31.47 & 35.41 \\
\cline { 2 - 4 } & MMSIM (\%) & 80.92 & 94.24 \\
\hline
\end{tabular}

Fig. 5: Cellule image compressed with Gall5/3 and SPIHT coding (a) original image (b) $R_{b}=0.4$, (c) $R_{b}=0.8$, (d) $R_{b}=1$

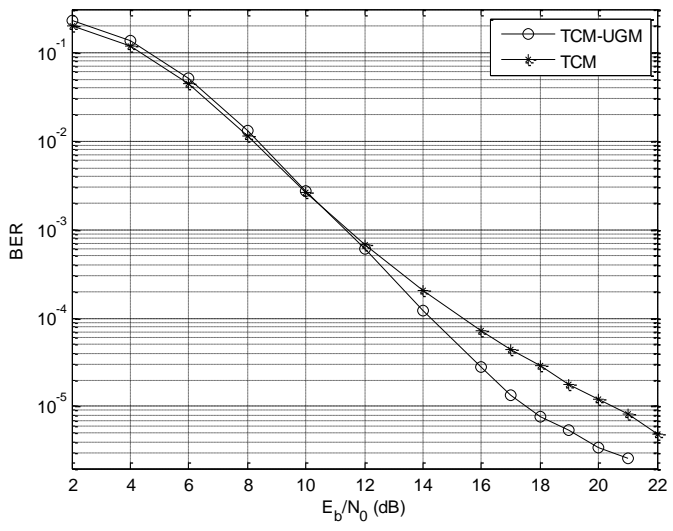

Fig. 6: BER performance of TCM and TCM-UGM schemes over Rayleigh fading channel

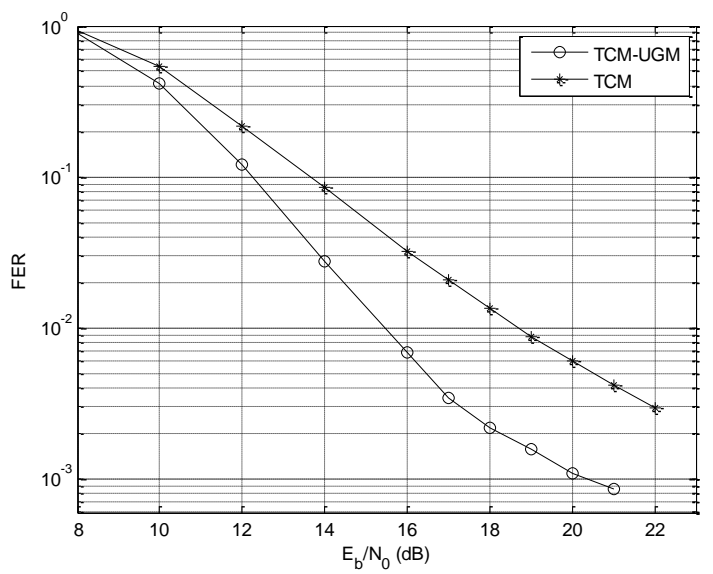

Fig. 7: FER performance of TCM and TCM-UGM schemes over Rayleigh fading channel

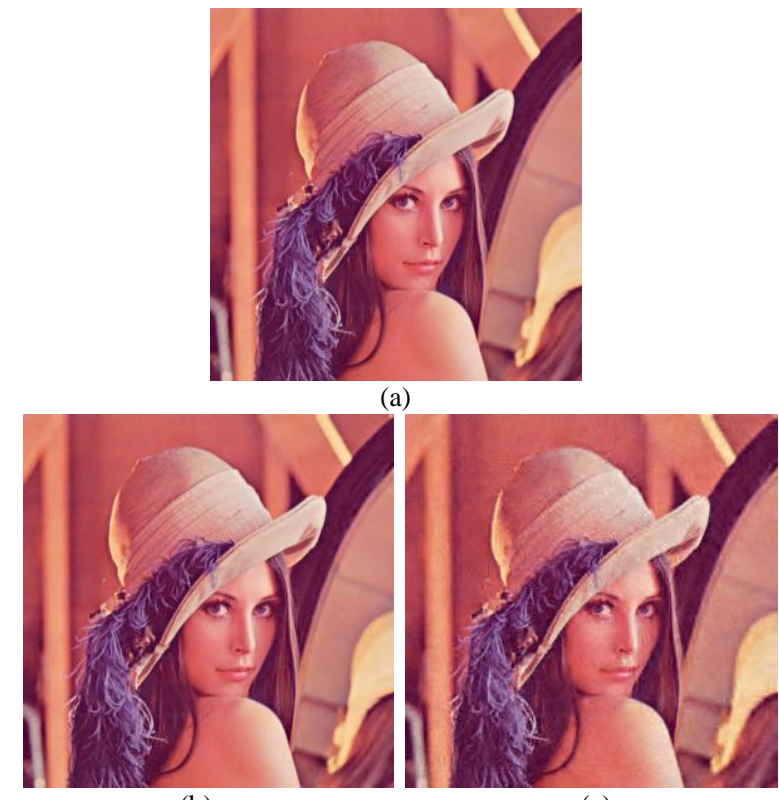

(b)

(c)

Fig. 8: (a) original image 'lena' (b) proposed TCM-UGM (c) TCM

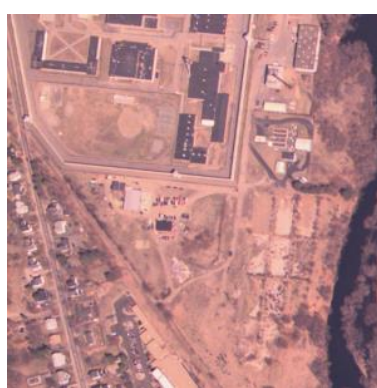

(a) 


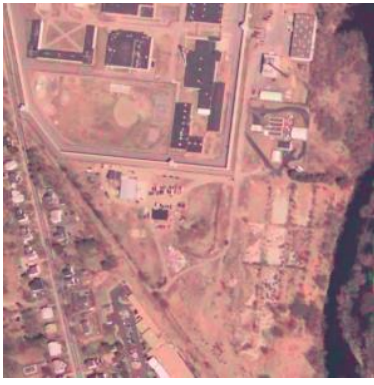

(b)

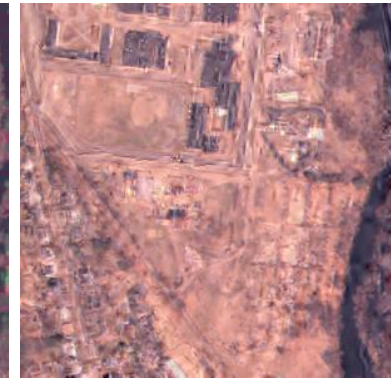

(c)
Fig. 9: (a) original image 'Concordaerial' (b) proposed TCM-UGM (c) TCM

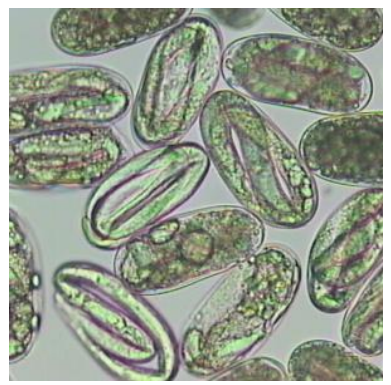

(a)

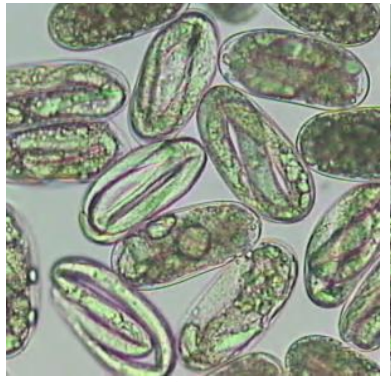

(b)

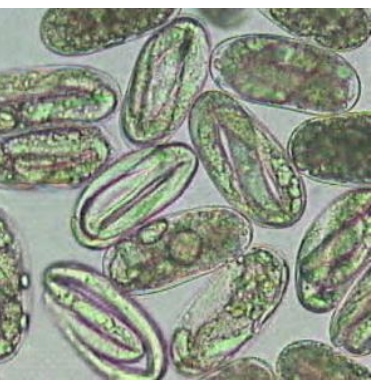

(c)
Fig. 10: (a) original image 'Cellule' (b) proposed TCM-UGM (c) TCM

\section{CONCLUSION}

In this work, the performance comparison of the variant TCM-UGM and the TCM was presented for spectral efficiency of $2 \mathrm{bits} / \mathrm{s} / \mathrm{Hz}$ for the compressed color image. The obtained results have shown that TCM-UGM present better performance in sense of BER and FER. The simulation is realized using 16-state TCM-UGM encoder, where the system gives the best result. A comparison between the TCM and the variant of the TCM-UGM for the compressed color image by the Gall $5 / 3$ and the SHIPT coding was performed. The two parameters of image quality used in the evaluation of image quality showed that the variant of 16-state TCMUGM reduces transmission errors and better protects the compressed image during transmission.

\section{REFERENCES}

[1] G. Ungerboeck, "Channel coding with multilevel/phase signals," IEEE Transactions on Information Theory, vol. 28, pp. 55-67, January 1982.
[2] A. Bassou and A. Djebbari, "Contribution to the Improvement of the Performance of Trellis-Coded Modulation," WSEAS Transactions on Communications, Vol. 6, No. 2, 2007, pp. 307-311.

[3] M. BENAISSA, A. Bassou, M. Beladgham, A. TalebAhmed, A. Moulay Lakhdar, 'Investigation of the performance of modified TCM scheme for the protection of SPIHT-based compressed images over fading channel', International Review on computers and Software (IRECOS), Vol. $8 \mathrm{~N}^{\circ} 1$ January 2013, pp. 271-276, doi:10.4236/ijens.2011.46046.

[4] V. Chappelier, Progressive Coding of Images by Directed Wavelet, Phd Thesis, Rennes 1 University, 2005.

[5] S. Mallat, "Multifrequency Channel Decompositions of Images and Wavelet Models," IEEE Transaction in Acoustic, Speech and Signal Processing, Vol. 37, No. 12, Dec. 1989, pp. 2091-2110.

[6] J.M. Shapiro, "Embedded Image Coding using Zerotrees of Wavelet Coefficients," IEEE Trans. Signal Processing, Vol. 41, No 12, Dec. 1993, pp. 3445 - 3462.

[7] A. Said, W.A. Pearlman, "A New, Fast, and Efficient Image Codec Based on Set Partitioning in Hierarchical Trees," IEEE Trans. Circuits Syst. Video Technol., Vol. 6, No. 3, June 1996, pp. 243-250.

[8] M. benaïssa, A. Bassou, M. Beladgham, A. Taleb-Ahmed, A. Moulay Lakhdar. "Investigation of the performance of modified TCM scheme for the protection of SPIHT-based compressed images over fading channel", International Review on computers and Software (IRECOS), Vol. 8 No.1, pp. 271-276, Jan. 2013.

[9] Antonini, M., Barlaud, M., Mathieu, P., and Daubechies, I., "Image Coding Using Wavelet Transform," IEEE Transactions Image Process, Vol. 1, No. 2, pp. 205-220, 1992.

[10] J.D. Villasenor, B. Belzer, J. Liao, "Wavelet Filter Evaluation for Image Compression," IEEE Transactions on Image Processing, Vol. 4, No. 8, Aug. 1995, pp.1053-1060.

[11] D.S. Taubman, M.W. Marcellin, JPEG2000: Image Compression Fundamentals, Standards and Practice, Kluwer Academic Publishers, London, 2002.

[12] H.R. Sheikh, M.F. Sabir, and A.C. Bovik, "A statistical evaluation of recent full reference image quality assessment algorithms," IEEE Trans. Image Processing, 15(11), pp. 3440-3451, Nov. 2006.

[13] A.B. Watson, L.B. Kreslake. "Measurement of Visual Impairment Scales for Digital Video", Human Vision and Electronic Imaging Conference, San Jose, CA, USA, SPIE Vol. 4299, Jan. 2001, 2001, pp. 79

[14] www.GE Med ical System.com (database).

[15] M. Beladgham, Y. Habchi, A. Moulay Lakhdar and A. Taleb-Ahmed. "A Comparative Study between Bandelet and Wavelet Transform Coupled by EZW and SPIHT Coder for Image Compression", International Journal of Image, Graphics and Signal Processing, Vol.5, No.12, pp.9-17, oct. 2013.

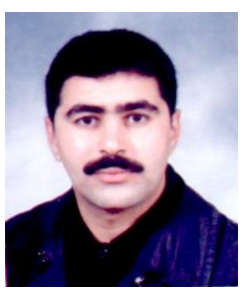

Mohamed BENAISSA obtained the Engineer degree in Electronics from university of Tlemcen, Algeria, and then a Magister in signals and systems from university of Tlemcen, Algeria and his Doctoral degree Es-Science from the University of Bechar, Algeria in 2013. Actually, $\mathrm{He}$ is an Associate Professor at University of Bechar, Algeria. His main interests are digital 


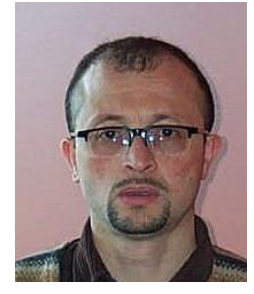

signal processing, Image processing, Medical image compression, turbo encoding schemes and iterative decoding over fading channels and wavelets transform. Algeria. He received the Dipl.El.-Ing. degree from the University of Tlemcen, Algeria in 1997, his Master from the University of Sidi Bel Abbes, Algeria in 2000, and his Doctoral degree Es-Science from the University of Sidi Bel Abbes, Algeria in 2006. Actually, $\mathrm{He}$ is an Associate Professor at University of Bechar, Algeria. His main interests are digital signal processing, turbo encoding schemes and iterative decoding over fading channels, and channel equalization.

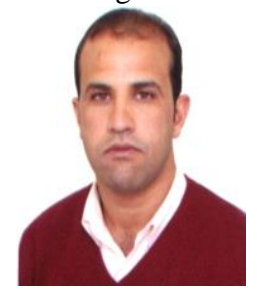

Mohammed BELADGHAM was born in Tlemcen, Algeria. He received the dipl. El.-Ing.from the university of Tlemcen, Algeria, and then a Master in signals and systems from the university of Tlemcen, Algeria. Since December 2012, he graduated PhD Es Sciences at the university of Tlemcen. His research interests are Image processing, Medical image compression, wavelets transform and optimal encoder.

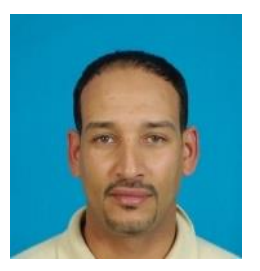

Abdelmalik TALEB-AHMED was born in Roubaix, France, in 1962. He received a Ph.D. in Electronics and Microwaves from the University de Lille 1 in 1992. From 1992 to 2004, He was an Associate Professor at the University du Littoral, Calais. Since 2004, He is currently a Professor at the University of Valenciennes, and does his research at the LAMIH UMR CNRS 8201 UVHC, His research interests includes signal and image processing: Image segmentation, Prior knowledge integration in image analysis, Partial Differential Equations and Variational Methods in image analysis, Multimodal signal processing, Medical image analysis, including multimodal image registration.

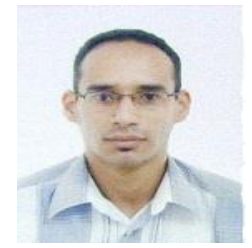

Abdelmounaim Moulay Lakhdar.I got my Engineering Degree in Telecommunication in 2000 at the Institute of Telecommunications in Oran. Magister was my second degree in Signal and telecom at Djillali LIABES university of Sidi Bel Abbes in 2003. From 2004 to this day I work in the Bechar University as lecturer. Since May 2009, I graduated PhD Es Sciences at the of Sidi Bel Abbes. I do my research at the Bechar University and Communications, Architecture and Media Laboratory (CAMR) (Djillali LIABES University). His research interests are Image transmission, Image processing, and digital transmission performances.

How to cite this paper: Benaïssa Mohamed, Bassou Abdesselam, Beladgham Mohammed, Taleb-Ahmed Abdelmalik, Moulay Lakhdar Abdelmounaim ,"Application of 16-State TCM-UGM and TCM for Improving the Quality of Compressed Color Image Transmission", IJIGSP, vol.6, no.10, pp.10-17, 2014.DOI: 10.5815/ijigsp.2014.10.02 